\title{
OSCILLATIONS OF HIGHER ORDER NEUTRAL DIFFERENTIAL EQUATIONS
}

\author{
JURANG YAN
}

(Received 25 February 1997; revised 18 July 1997)

Communicated by J. R. J. Groves

\begin{abstract}
Some new oscillation criteria for higher order neutral functional differential equations of the form

$$
\frac{d^{\prime \prime}}{d t^{\prime \prime}}(x(t)+c x(t-h)+\bar{c} x(t+\bar{h}))+q x(t-g)+p x(t+\bar{g})=0,
$$

$n$ is even, are established.

1991 Mathematics subject classification (Amer. Math. Soc.): 34K15.

Keywords and phrases: Oscillation, neutral differential equation, characteristic equation.
\end{abstract}

\section{Introduction}

This paper deals with the even order neutral functional differential equation

$(E)(x(t)+c x(t-h)+\bar{c} x(t+\bar{h}))^{(n)}+q x(x-g)+p x(t+\bar{g})=0, \quad n$ even,

where $c, \bar{c}, h, \bar{h}, p$ and $q$ are real numbers, $g$ and $\bar{g}$ are positive constants.

Recently, the oscillatory behavior of solutions of $(E)$ has been studied by Grace [2]. The purpose of this paper is to obtain some new easily verifiable sufficient conditions, involving only the coefficients and the arguments, under which all solutions of $(E)$ are oscillatory. Our technique, differing greatly from that in [2], is based on the study of the characteristic equation

$$
\lambda^{n}\left(1+c e^{-\lambda h}+\bar{c} e^{\lambda \bar{h}}\right)+q e^{-\lambda g}+p e^{\lambda \bar{s}}=0 .
$$

Reseach was partially supported by the Natural Science Foundation of Shanxi Province (C) 1998 Australian Mathematical Society $0263-6115 / 98 \$ A 2.00+0.00$ 
Necessary and sufficient conditions (in terms of the characteristic equations) for the oscillations of all solutions of higher order neutral differential equations have been established by Bilchev, Grammatikopoulos and Stavroulakis [1], Ladas, Partheniadis and Sficas [3] and Wang [5]. The oscillation criteria obtained in this paper improve noticeably the results in [2] and [4].

Let $t_{0} \geq 0$. By a solution of $(E)$ we mean a continuous function $x$ defined on the interval $\left[t_{0}-\tau, \infty\right)$, where $\tau=\max \{h, g\}$ such that $x(t)+c x(t-h)+\bar{c} x(t+\bar{h})$ is $n$-times continuously differentiable for all $t \geq t_{0}$. As is customary, a solution is called oscillatory if it has arbitrarily large zeros. Otherwise, the solution is called non-oscillatory. Equation $(E)$ is called oscillatory if all of its solutions are oscillatory.

\section{Main results}

The oscillation criteria in this paper are based on the following lemma which is extracted from results in [1] and [5].

LEMMA. A necessary and sufficient condition for the oscillation of $(E)$ is that its characteristic equation $\left(E^{*}\right)$ has no real roots.

Like Grace [2], we study the oscillatory character of the following equations, as some special cases of $(E)$,

$\begin{array}{ll}\left(E_{1}\right) & \frac{d^{\prime \prime}}{d t^{\prime \prime}}(x(t)+c x(t-h)-\bar{c} x(t+\bar{h}))=q x(t-g)+p x(t+\bar{g}), \\ \left(E_{2}\right) & \frac{d^{n}}{d t^{\prime \prime}}(x(t)+c x(t+h)-\bar{c} x(t+\bar{h}))=q x(t-g)+p x(t+\bar{g}) . \\ \left(E_{3}\right) & \frac{d^{\prime \prime}}{d t^{\prime \prime}}(x(t)+c x(t-h)+\bar{c} x(t+\bar{h}))=q x(t-g)+p x(t+\bar{g}),\end{array}$

and

(E) $\quad \frac{d^{n}}{d t^{n}}(x(t)-c x(t-h)-\bar{c} x(t+\bar{h}))+q x(t-g)+p x(t+\bar{g})=0$.

where $n$ is even, $c, \bar{c}, g, h$ and $\bar{h}$ are non-negative, $g, \bar{g}, p$ and $q$ are positive real numbers. The characteristic equations of $\left(E_{1}\right)-\left(E_{4}\right)$ are respectively

$\left(E_{1}^{*}\right)$

$\left(E_{2}^{*}\right)$

$\left(E_{3}^{*}\right)$

$$
\begin{aligned}
& F_{1}(\lambda):=\lambda^{\prime \prime}\left(1+c e^{-\lambda h}-\bar{c} e^{\lambda \bar{h}}\right)-q e^{-\lambda g}-p e^{\lambda \bar{g}}=0, \\
& F_{2}(\lambda):=\lambda^{\prime \prime}\left(1+c e^{\lambda h}-\bar{c} e^{\lambda \bar{h}}\right)-q e^{-\lambda g}-p e^{\lambda \bar{g}}=0, \\
& F_{3}(\lambda):=\lambda^{\prime \prime}\left(1+c e^{-\lambda h}+\bar{c} e^{\lambda \bar{h}}\right)-q e^{-\lambda g}-p e^{\lambda \bar{g}}=0
\end{aligned}
$$


and

$\left(E_{4}^{*}\right) \quad F_{4}(\lambda):=\lambda^{\prime \prime}\left(1-c e^{-\lambda h}-\bar{c} e^{\lambda \bar{h}}\right)+q e^{-\lambda g}+p e^{\lambda \bar{s}}=0$.

First of all, we consider $\left(E_{1}\right)$ and establish the following result for its oscillatory character.

THEOREM 1. If

$$
p \bar{g}\left(\frac{e}{n}\right)^{n}+\bar{c} \exp \left(\left[\frac{p}{1+c}\right]^{1 / n} \bar{h}\right)>1+c
$$

and

$$
q(g-h)^{n}\left(\frac{e}{n}\right)^{n}>1+c \text { and } g>h
$$

then $\left(E_{1}\right)$ is oscillatory.

ProOF. For $\lambda \neq 0$, from $\left(E_{1}^{*}\right)$, we have that

$$
-F_{1}(\lambda) / \lambda^{n}=\left(q e^{-\lambda, g}+p e^{\lambda \bar{s}}\right) / \lambda^{n}-\left(1+c e^{-\lambda h}-\bar{c} e^{\lambda \bar{h}}\right)
$$

and

$$
-F_{1}(\lambda) e^{\lambda h} / \lambda^{\prime \prime}=\left(q e^{-\lambda(g-h)}+p e^{\lambda(\bar{g}+h)}\right) / \lambda^{n}-\left(e^{\lambda h}+c-\bar{c} e^{\lambda(\bar{h}+h)}\right) .
$$

Now, our strategy is to prove that $F_{1}(\lambda)<0$ for all $\lambda \in(-\infty, \infty)$ in each of the following four cases.

Case 1. $0<\lambda \leq(p /(1+c))^{1 / n}$. From (3), in this case we have

$$
-F_{1}(\lambda) / \lambda^{n}>\left(q e^{-i \mu}+p e^{\lambda \bar{s}}\right) / \lambda^{n}-(1+c)>p /\left(\frac{p}{1+c}\right)-(1+c)=0 .
$$

Thus $F_{1}(\lambda)<0$ for $0<\lambda \leq(p /(1+c))^{1 / n}$.

Case 2. $\lambda>(p /(1+c))^{1 / n}$. In view of $(1)$ and the inequality $e^{x} \geq e x$ for all $x \geq 0$,

(3) yields

$$
\begin{aligned}
-F_{1}(\lambda) / \lambda^{n} & \geq q e^{-\dot{\lambda} q} / \lambda^{\prime \prime}+p \bar{g}^{n}\left(e^{\frac{i}{n} \bar{g}} / \frac{\lambda}{n} \bar{g}\right)^{n} \frac{1}{n^{n}}-(1+c)+\bar{c} \exp \left(\left[\frac{p}{1+c}\right]^{1 / n} \bar{h}\right) \\
& >p \bar{g}^{n}\left(\frac{e}{n}\right)^{\prime \prime}-(1+c)+\bar{c} \exp \left(\left[\frac{p}{1+c}\right]^{1 / n} \bar{h}\right)>0,
\end{aligned}
$$

which implies that $F_{1}(\lambda)<0$ for $\lambda>(p /(1+c))^{1 / n}$. 
Case 3. $\lambda<0$. By using (2) and the inequality $e^{x} \geq e x$ for $x \geq 0$, from (4) we obtain

$$
\begin{aligned}
-F_{1}(\lambda) e^{\lambda h} / \lambda^{n} & \geq q(g-h)^{n}\left[e^{-\lambda(g-h) / n} / \lambda(g-h) / n\right]^{n} n^{-n}+p e^{\lambda(\bar{g}+h)} / \lambda^{n}-\left(e^{\lambda h}+c\right) \\
& \geq q(g-h)^{n}(e / n)^{n}-(1+c)>0 .
\end{aligned}
$$

Therefore, in this case $F_{1}(\lambda)<0$ for $\lambda<0$.

Cases $1-3$ and $F_{1}(0)<0$ imply that $F_{1}(\lambda)<0$ for $\lambda \in(-\infty, \infty)$, that is, $\left(\mathrm{E}_{1}^{*}\right)$ has no real roots. By using the lemma, we conclude that $\left(E_{1}\right)$ is oscillatory. This completes the proof.

REMARK 1. Theorem 1 improves Theorem 1 of Grace [2].

Next, we present a theorem which describes the oscillatory character of $\left(E_{2}\right)$.

THEOREM 2. If

$$
p(\bar{g}-h)^{n}(e / n)^{n}+\bar{c}>1+c \text { and } \bar{g}>h, \bar{h}>h,
$$

and

$$
q g^{n}\left(\frac{e}{n}\right)^{n}+\bar{c} \exp \left(-\left[\frac{q}{1+c}\right]^{1 / n} \bar{h}\right)>1+c,
$$

then $\left(E_{2}\right)$ is oscillatory.

PrOOF. From the characteristic equation $\left(E_{2}^{*}\right)$ of $\left(E_{2}\right)$, for $\lambda \neq 0$, we get

$$
-F_{2}(\lambda) / \lambda^{n}=\left(q e^{-\lambda g}+p e^{\lambda \bar{s}}\right) / \lambda^{n}-\left(1+c e^{i, h}-\bar{c} e^{\lambda \bar{h}}\right),
$$

and

$$
-F_{2}(\lambda) e^{-\lambda h} / \lambda^{n}=\left(q e^{-\lambda(g+h)}+p e^{\lambda(\bar{g}-h)}\right) / \lambda^{n}-\left(e^{-\lambda h}+c-\bar{c} e^{\lambda(\bar{h}-h)}\right) .
$$

As in the proof of Theorem 1, we consider the following four cases.

Case 1. $0<\lambda \leq(p /(1+c))^{1 / n}$. From (8) it follows that

$$
-F_{2}(\lambda) e^{-\lambda h} / \lambda^{n}>p e^{\lambda(\bar{g}-h)} /(p /(1+c))-(1+c)>0 .
$$

which implies $F_{2}(\lambda)<0$ for $0<\lambda \leq(p /(1+c))^{1 / n}$. 
Case 2. $\lambda>(p /(1+c))^{1 / n}$. Then from (8), (5) and the fact that $e^{x} \geq e x$ for $x \geq 0$, we have

$$
\begin{aligned}
-F_{2}(\lambda) e^{-\lambda h} / \lambda^{n} & \geq p(\bar{g}-h)^{n}\left(e^{\lambda(\bar{g}-h) / n} /[\lambda(\bar{g}-h) / n]\right)^{n} n^{-n}-(1+c-\bar{c}) \\
& \geq p(\bar{g}-h)^{n}(e / n)^{n}-(1+c-\bar{c})>0,
\end{aligned}
$$

Hence, in this case, $F_{2}(\lambda)<0$.

Case 3. $-(q /(1+c))^{1 / n} \leq \lambda<0$. From (7) and (6) we get

$$
-F_{2}(\lambda) / \lambda^{n} \geq q g^{n}\left(e^{-\lambda g / n} /(-\lambda g / n)^{n} n^{-n}-\left(1+c-\bar{c} \exp \left[-(q /(1+c))^{1 / n} \bar{h}\right]\right)>0,\right.
$$

that is, $F_{2}(\lambda)>0$ for $-(q /(1+c))^{1 / n} \leq \lambda<0$.

Case 4. $\lambda<-(q /(1+c))^{1 / n}$. In this case, using (7) we observe

$$
-F_{2}(\lambda) / \lambda^{n} \geq q e^{-\lambda g} / \frac{q}{1+c}-(1+c)>0
$$

which implies $F_{2}(\lambda)<0$ for $\lambda<-(q /(1+c))^{1 / n}$.

From cases $1-4$ and $F_{2}(0)<0$, we see that for all $\lambda \in(-\infty, \infty), F_{2}(\lambda)<0$. By the Lemma, we conclude that $\left(E_{2}\right)$ is oscillatory, and the proof of the theorem is complete.

REMARK 2. Our Theorem 2 improves Theorem 2 in [2].

The following result is concerned with the oscillatory behavior of $\left(E_{3}\right)$.

THEOREM 3. If

(9)

$$
p(\bar{g}-\bar{h})^{n}\left(\frac{e}{n}\right)^{n}>1+\bar{c}+c \exp \left[-\left(\frac{p}{1+c+\bar{c}}\right)^{1 / n}(h+\bar{h})\right], \quad \text { and } \bar{g}>\bar{h},
$$

and

(10)

$$
q(g-h)^{n}\left(\frac{e}{n}\right)^{n}>1+c+\bar{c} \exp \left[-\left(\frac{q}{1+c+\bar{c}}\right)^{1 / n}(\bar{h}+h)\right], \quad \text { and } g>h,
$$

then $\left(E_{3}\right)$ is oscillatory.

ProOF. From $\left(E_{3}^{*}\right)$, for $\lambda \neq 0$ we obtain

$$
-F_{3}(\lambda) e^{-\lambda \bar{h}} / \lambda^{n}=\left(q e^{-\lambda(g+\bar{h})}+p e^{\lambda(\bar{g}+\bar{h})}\right) / \lambda^{n}-\left(e^{-\lambda \bar{h}}+c e^{-\lambda(h+\bar{h})}+\bar{c}\right)
$$


and

$$
-F_{3}(\lambda) e^{\lambda h} / \lambda^{n}=\left(q e^{-\lambda(g-h)}+p e^{\lambda(\bar{g}+h)}\right) / \lambda^{n}-\left(e^{\lambda h}+c+\bar{c} e^{\lambda(h+\bar{h})}\right) .
$$

Now, consider the following four cases.

Case 1. $0<\lambda \leq(p /(1+c+\bar{c}))^{1 / n}$. From (11), we get

$$
-F_{3}(\lambda) e^{-\lambda \bar{h}} / \lambda^{n}>p /(p /(1+c+\bar{c}))-(1+c+\bar{c})=0 .
$$

Case 2. $\lambda>(p /(1+c+\bar{c}))^{1 / n}$. In view of (11) and (9) we obtain

$$
\begin{aligned}
-F_{3}(\lambda) e^{-\lambda \bar{h}} / \lambda^{n}> & p(\bar{g}-\bar{h})^{n}\left(\frac{e}{n}\right)^{n} \\
& -\left[1+c \exp \left(-\left[\frac{p}{1+c+\bar{c}}\right]^{1 / n}(h+\bar{h})\right)+\bar{c}\right]>0 .
\end{aligned}
$$

Case 3. $-(q /(1+c+\bar{c}))^{1 / n} \leq \lambda<0$. From (12) it follows that

$$
-F_{3}(\lambda) e^{\lambda h} / \lambda^{n}>q / \frac{q}{1+c+\bar{c}}-(1+c+\bar{c})=0 .
$$

Case 4. $\lambda<-(q /(1+c+\bar{c}))^{1 / n}$. Then, from (12) and (10) we have

$$
\begin{aligned}
-F_{3}(\lambda) e^{\lambda h} / \lambda^{n} \geq & q(g-h)^{n}\left(\frac{e}{n}\right)^{n} \\
& -\left(1+c+\bar{c} \exp \left[-\left(\frac{q}{1+c+\bar{c}}\right)^{1 / n}(\bar{h}+h)\right]\right)>0 .
\end{aligned}
$$

From cases $1-4$ and $F_{3}(0)<0$, we observe $F_{3}(\lambda)<0$ for all $\lambda \in(-\infty, \infty)$. By the lemma, $\left(E_{3}\right)$ is oscillatory, and the proof of the theorem is complete.

REMARK 3. Theorem 3 improves Theorem 3 in [2].

Finally, we present an oscillation criterion for $\left(E_{4}\right)$.

THEOREM 4. If $c+\bar{c}>0$,

$$
p(\bar{g}-\bar{h})^{n}\left(\frac{e}{n}\right)^{n}>c \exp \left[-\left(\frac{p}{c+\bar{c}}\right)^{1 / n}(h+\bar{h})\right]+\bar{c} \text { and } \bar{g}>\bar{h}
$$

and

(14) $q(g-h)^{n}\left(\frac{e}{n}\right)^{n}>c+\bar{c} \exp \left[-\left(\frac{q}{c+\bar{c}}\right)^{1 / n}(\bar{h}+h)\right]$ and $g>h$,

then $\left(E_{4}\right)$ is oscillatory. 
PROOF. By the characteristic equation $\left(E_{4}^{*}\right)$ of $\left(E_{4}\right)$ we obtain

$$
F_{4}(\lambda) e^{-\lambda \bar{h}} / \lambda^{n}=\left[q e^{-\lambda(g+\bar{h})}+p e^{\lambda(\bar{g}-\bar{h})}\right] / \lambda^{n}+e^{-\lambda \bar{h}}-c e^{-\lambda(h+\bar{h})}-\bar{c}
$$

and

$$
F_{4}(\lambda) e^{\lambda h} / \lambda^{n}=\left[q e^{-\lambda(g-h)}+p e^{\lambda(\bar{g}+h)}\right] / \lambda^{n}+e^{\lambda h}-c-\bar{c} e^{\lambda(\bar{h}+h)}
$$

Consider the following four cases.

Case $1.0<\lambda \leq(p /(c+\bar{c}))^{1 / n}$. From (15) we have

$$
F_{4}(\lambda) e^{-i \bar{h}} / \lambda^{n} \geq p / \frac{p}{c+\bar{c}}+e^{-\lambda \bar{h}}-c-\bar{c}>0 .
$$

Case 2. $\lambda>(p /(c+\bar{c}))^{1 / n}$. In this case, from (15) and (13), it follows that

$$
F_{4}(\lambda) e^{-\lambda \bar{h}} / \lambda^{n} \geq p(\bar{g}-\bar{h})^{n}\left(\frac{e}{n}\right)^{n}-c \exp \left[-\left(\frac{p}{c+\bar{c}}\right)^{1 / n}(h+\bar{h})\right]-\bar{c}>0 .
$$

Case 3. $-(q /(c+\bar{c}))^{1 / n} \leq \lambda<0$. From (16) we get

$$
F_{4}(\lambda) e^{i, h} / \lambda^{n} \geq q / \frac{q}{c+\bar{c}}+e^{\lambda h}-c-\bar{c}>0 .
$$

Case 4. $\lambda<-(q /(c+\bar{c}))^{1 / n}$. By using (16) and (14) we obtain

$$
F_{4}(\lambda) e^{\lambda h} / \lambda^{n} \geq q(g-h)^{n}\left(\frac{e}{n}\right)^{n}-c-\bar{c} \exp \left[-\left(\frac{q}{c+\bar{c}}\right)^{1 / n}(h+\bar{h})\right]>0 .
$$

From cases $1-4$ and $F_{4}(0)>0$, it follows that $F_{4}(\lambda)>0$ for all $\lambda \in(-\infty, \infty)$ which means that $\left(E_{4}\right)$ is oscillatory, and the proof of the theorem is complete.

REMARK 4. Theorem 4 improves Theorem 4 in [2] and the theorem in [4].

The following examples are illustrative.

EXAMPLE 1. Consider the differential equation

$$
[x(t)+x(t-2 \pi)-2 x(t+2 \pi)]^{(12)}=2 \sin (t-8 \pi)+2 \sin (t+\pi) .
$$

It is easy to check that conditions (1) and (2) are satisfied, and hence (17) is oscillatory and $x(t)=\sin t$ and $x(t)=\cos t$ are two solutions of (17). 
EXAMPLE 2. Consider the differential equation

(18)

$$
\left[x(t)-e^{4} x\left(t-\frac{\pi}{2}\right)-e^{4} x\left(t+\frac{\pi}{2}\right)\right]^{(4)}+3 x\left(t-\frac{3 \pi}{2}\right)+2 x\left(t+\frac{3}{2} \pi\right)=0 .
$$

The conditions (13) and (14) are satisfied and hence (18) is oscillatory. One such solution is $x(t)=\cos t$.

REMARK 5. In the above examples we observe that conditions (1) and (22) of Theorems 1 and 4 in [2], respectively, are violated. Hence Theorems 1 and 4 in [2] fail to apply to (17) and (18) and we believe that the oscillatory behavior of (17) and (18) is not deducible from known oscillation criteria.

From the proofs of Theorems $1-4$ and the lemma, it is easy to prove the following results.

COROLLARY 1. If condition (1) (respectively (5), (9) or (13)) is satisfied, then every solution $x(t)$ of $\left(E_{1}\right)$ (respectively $\left(E_{2}\right),\left(E_{3}\right)$ or $\left(E_{+}\right)$) is either oscillatory or else $x^{(j)}(t) \rightarrow 0$ as $t \rightarrow \infty, j=0,1, \ldots, n-1$.

COROLLARY 2. If only condition (2) (respectively (6), (10) or (14)) is satisfied, then every solution $x(t)$ of $\left(E_{1}\right)$ (respectively $\left(E_{2}\right),\left(E_{3}\right)$ or $\left(E_{4}\right)$ ) is either oscillatory or else $x^{(j)}(t) \rightarrow \infty$ as $t \rightarrow \infty, j=0,1, \ldots, n-1$.

REMARK 6. Our technique can be extended to odd order neutral functional differential equations.

\section{Acknowledgement}

I am grateful to the referee for several valuable suggestions.

\section{References}

[1] S. J. Bilchev, M. K. Grammatikopoulos and I. P. Stavroulakis, 'Oscillations of higher order neutral differential equations', J. Austral. Math. Soc. (Series A) 52 (1992).

[2] S. R. Grace, 'Oscillation criteria for $n$th order neutral functional equations', J. Math. Anal. Appl. 184 (1994), 44-55.

[3] G. Ladas, E. C. Partheniadis and Y. G. Sficas, 'Necessary and sufficient conditions for oscillations of second order neutral equations', J. Math. Anal. Appl. 138 (1989), 214-231. 
[4] G. Ladas and Y. G. Sficas, 'Oscillations of higher order neutral equations', J. Austral. Math. Soc. (Series B) 27 (1986), 502-517.

[5] Z. Wang, 'A necessary and sufficient condition for the oscillation of higher order neutral equations', Tohoku Math. J. 41 (1989), 575-588.

Department of Mathematics

Shanxi University

Taiyuan Shanxi 030006

People's Republic of China 